\title{
Phylogenomic exploration of the relationships between strains of Mycobacterium avium subspecies paratuberculosis
}

Josephine M. Bryant ${ }^{1,2}$, Virginie C. Thibault ${ }^{3}$, David G. E. Smith ${ }^{3,4}$, Joyce McLuckie ${ }^{3}$, lan Heron³ Iker A. Sevilla $^{5}$, Franck Biet ${ }^{6}$, Simon R. Harris ${ }^{1}$, Duncan J. Maskell ${ }^{7}$, Stephen D. Bentley ${ }^{1}$, Julian Parkhill ${ }^{1}$ and Karen Stevenson ${ }^{3^{*}}$

\begin{abstract}
Background: Mycobacterium avium subspecies paratuberculosis (Map) is an infectious enteric pathogen that causes Johne's disease in livestock. Determining genetic diversity is prerequisite to understanding the epidemiology and biology of Map. We performed the first whole genome sequencing (WGS) of 141 global Map isolates that encompass the main molecular strain types currently reported. We investigated the phylogeny of the Map strains, the diversity of the genome and the limitations of commonly used genotyping methods.

Results: Single nucleotide polymorphism (SNP) and phylogenetic analyses confirmed two major lineages concordant with the former Type $S$ and Type $C$ designations. The Type I and Type III strain groups are subtypes of Type S, and Type B strains are a subtype of Type $C$ and not restricted to Bison species.

We found that the genome-wide SNPs detected provided greater resolution between isolates than currently employed genotyping methods. Furthermore, the SNP used for IS1311 typing is not informative, as it is likely to have occurred after Type $\mathrm{S}$ and $\mathrm{C}$ strains diverged and does not assign all strains to the correct lineage. Mycobacterial Interspersed Repetitive Unit-Variable Number Tandem Repeat (MIRU-VNTR) differentiates Type S from Type C but provides limited resolution between isolates within these lineages and the polymorphisms detected do not necessarily accurately reflect the phylogenetic relationships between strains.

WGS of passaged strains and coalescent analysis of the collection revealed a very high level of genetic stability, with the substitution rate estimated to be less than 0.5 SNPs per genome per year.

Conclusions: This study clarifies the phylogenetic relationships between the previously described Map strain groups, and highlights the limitations of current genotyping techniques. Map isolates exhibit restricted genetic diversity and a substitution rate consistent with a monomorphic pathogen. WGS provides the ultimate level of resolution for differentiation between strains. However, WGS alone will not be sufficient for tracing and tracking Map infections, yet importantly it can provide a phylogenetic context for affirming epidemiological connections.
\end{abstract}

Keywords: Mycobacterium avium subspecies paratuberculosis, Johne's disease, Genome wide sequencing, Phylogenomics, Genotyping

\footnotetext{
* Correspondence: karen.stevenson@moredun.ac.uk

${ }^{3}$ Moredun Research Institute, Pentlands Science Park, Penicuik EH26 OPZ, UK
}

Full list of author information is available at the end of the article

\section{Biomed Central}

(C) 2015 Bryant et al. Open Access This article is distributed under the terms of the Creative Commons Attribution 4.0 International License (http://creativecommons.org/licenses/by/4.0/), which permits unrestricted use, distribution, and reproduction in any medium, provided you give appropriate credit to the original author(s) and the source, provide a link to the Creative Commons license, and indicate if changes were made. The Creative Commons Public Domain Dedication waiver (http://creativecommons.org/publicdomain/zero/1.0/) applies to the data made available in this article, unless otherwise stated. 


\section{Background}

Mycobacterium avium subspecies paratuberculosis (Map) is an enteric pathogen causing Johne's disease, which is responsible for considerable economic losses to the livestock and associated industries on a global scale [1]. Map can infect a broad range of host species [2-4], but clinical disease is reported only in ruminants [5], camelids [6, 7], rabbits [8] and hares [9]. Map has been detected in humans in a subset of patients with Crohn's disease [10]. Although the zoonotic potential of Map remains a controversial issue, its presence in the food chain is an important consideration for the food industry and there is a drive towards controlling the organism on the farm.

Understanding the genetic diversity of Map is important for both epidemiological and biological reasons and will inform the development of improved diagnostics and effective vaccines for controlling disease. However, like the related human pathogens Mycobacterium tuberculosis and Mycobacterium leprae, Map is genetically monomorphic [11] so presents a challenge to genotyping. Over the years, various molecular typing techniques have been used to differentiate between Map isolates (for reviews see [12, 13]). In 1990, Collins et al. [14] described two major groups of strains, which appeared to correlate with the host of origin and were designated "Sheep-type" (Type S) or "Cattle-type" (Type C). However, as strain typing was more widely applied, it became apparent that the correlation between strain type and host species was not absolute and it was not always clear when the 'sheep' or 'cattle' designation referred to the strain type or the host provenance. To avoid confusion, it was proposed that these strain types be referred to as Type I (Type S) or Type II (Type C) [15], although both designations are still in use. These two major strain groups can be differentiated based on their genotype, growth characteristics and pathogenesis $[13,16]$.

Other strain groups have been identified. A group of strains designated "Type III" has been described [17-19] and was suggested to represent an evolutionary intermediate between the two major strain groups. 'Bison' (Type B) strains comprise another group of strains. These strains were first isolated from bison (Bison bison) in Montana, USA and although molecular analysis characterized them as Type $C$, the unusual growth requirements of the isolates and clinical presentation in the infected animals suggested that they may be different from the strains commonly isolated from cattle [20]. Initially these strains were differentiated on the basis of the number of copies with a $\mathrm{C}$ or a $\mathrm{T}$ at base position 223 in the insertion sequence IS1311 [20]. Subsequent genotyping of more isolates from bison demonstrated that isolates obtained from bison in India were different from those from US bison and these have been referred to as
'Indian bison type' [21]. Sohal et al. [22] recently identified a unique TG deletion at base pair positions 64 and 65 of IS1311 at locus 2 in the Indian bison type strains.

Whilst these different strain groups have been defined using different typing procedures, the phylogenetic relationships between them have not been elucidated. In this study we undertake the first whole genome sequencing (WGS) study using a comprehensive international panel of strains to determine the evolution, population structure and phylogeography of Map. We undertake single nucleotide polymorphism (SNP) analysis of a panel of $141 \mathrm{Map}$ isolates representing 17 countries, nine host species and all of the strain groups described above to determine the extent of genetic diversity and phylogenetic relationships between the strains. In addition, we assess the performance of current typing techniques compared to the ultimate level of resolution obtained with WGS to assess their utility for epidemiological studies, surveillance and tracing of infections. Finally we investigate the stability of the genome following in vitro passage and provide an estimate for the Map mutation substitution rate.

\section{Methods}

\section{Panel of strains}

For this study, a panel of 141 Map isolates was carefully selected to maximize genetic diversity and include representative isolates for all strain groups identified to date. Details of the isolates comprising the panel are given in Additional file 1: Table S1. To maximize genetic diversity, isolates were chosen with different multiplex pulsed field gel electrophoresis (PFGE) profiles (total of 68) and selected from different geographical regions representing 17 countries (Argentina, Canada, Czech Republic, Faroe Islands, France, Germany, Greece, India, Ireland, Italy, The Netherlands, New Zealand, Norway, Spain, United Kingdom, United States of America and Venezuela). The isolates were from cattle, sheep, goats, deer (unspecified species), bison (Bison bison), buffalo (Bubalis bubalis), moufflon (Ovis musimon) and humans with Crohn's disease. Four vaccine strains and an environmental isolate from water were also included. In total, the panel comprised 20 Type S isolates, (of which 14 were Type I and six Type III), eight Type B isolates and the remaining 112 isolates were Type C. Twelve strains with identical genotypes as determined by a combination of PFGE and Mycobacterial Interspersed Repetitive Unit-Variable Number Tandem Repeat (MIRU-VNTR) typing were included to investigate the discriminatory power of WGS and multiple passages of two field strains and the reference strain MapK10 were included to investigate genome stability. All isolates were positive for IS900. PFGE was performed according to the standardized protocol published by Biet et al. [23]. MIRU-VNTR analysis was 


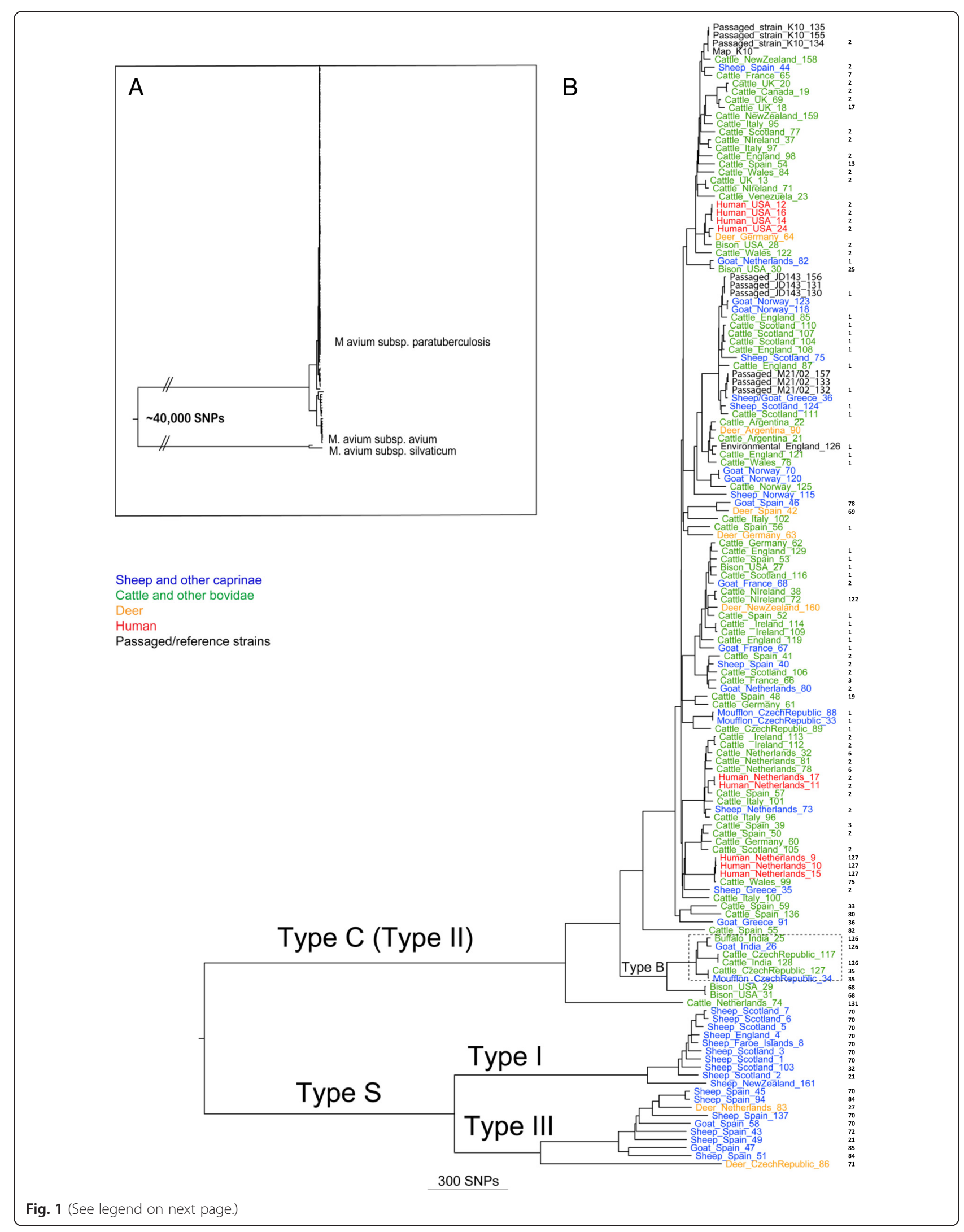


(See figure on previous page.)

Fig. 1 Whole genome SNP-based phylogenetic tree of strains included in this study. a Phylogenetic tree of Map and non-Map strains built using FastTree [52]. Map strains form a single clade and are separated from other mycobacterial species by at least 40,000 SNPs (branches shortened for illustrative purposes). $\mathbf{b}$ Maximum likelihood phylogenetic tree of Map strains sequenced as part of this study. The tree was created using RAxML [30], and is based on SNPs identified through mapping to Map-K10 as described in the text. Branches are annotated with the host, country of origin and isolate MAPMRI numbers. Numbers in black to the far right represent the INMV profiles. Previously described lineages are labeled. The dashed box represents strains designated as the Indian bison type. Bootstrap values are shown in Additional file 2: Figure S1

performed as described by Thibault et al. [24] and the profile number assigned by INRA and published in an online database [25]. The presence of $\operatorname{LSP}^{\mathrm{A}} 20$ (for Type $\mathrm{C}$ strains) and $\mathrm{LSP}^{\mathrm{A}} 4$-II (for Type $\mathrm{S}$ strains) was determined by PCR using primers reported by Semret et al. [26] and conditions described by Biet et al. [23]. Type I and III strains were assigned according to the SNP analysis of the gyrA and gyrB genes as described by Castellanos et al. [19] or according to their PFGE profile [15, 17]. SNP analysis of IS1311 by PCR restriction enzyme analysis to determine types $\mathrm{S}, \mathrm{C}$ and $\mathrm{B}$ was undertaken as described by Whittington et al. [20]. Two other isolates representing members of the Mycobacterium avium complex (Mycobacterium avium subspecies avium [Maa] and Mycobacterium avium subspecies silvaticum $[M a s])$ were included in the panel for WGS to place the Map strains in a wider mycobacterial context.

\section{Culture of isolates}

Isolates were received from participating laboratories growing on a variety of media and were subsequently propagated on 7H11+ agar (Middlebrook 7H11 supplemented with $20 \%$ [ $\mathrm{vol} / \mathrm{vol}]$ heat-inactivated newborn calf serum, $2.5 \%$ [vol/vol] glycerol, $2 \mathrm{mM}$ asparagine, $10 \%$ [vol/vol] Middlebrook oleic acid-albumin-dextrosecatalase [OADC] enrichment medium [Becton Dickinson, Oxford, Oxfordshire, United Kingdom], Selectatabs [code MS 24; MAST Laboratories Ltd., Merseyside, United Kingdom], and $2 \mu \mathrm{g} \mathrm{ml}^{-1}$ mycobactin J [Allied Monitor, Fayette, Mo.]). For preparation of DNA, the isolates were subcultured in Middlebrook 7H9 broth supplemented with $0.2 \%$ (vol/vol) glycerol, $0.05 \%$ (vol/ vol) Tween 80 and $2 \mu \mathrm{g} \mathrm{ml}^{-1}$ mycobactin $J$ and stirred during incubation at $37^{\circ} \mathrm{C}$.

For investigating genome stability, the two field strains M21/02 and JD143 and Map K10 were subcultured on $7 \mathrm{H} 11+$ agar every 6-12 weeks. The passage number was noted and glycerol stocks prepared and archived at $-80^{\circ}$ $\mathrm{C}$ for each passage.

\section{Preparation of DNA}

Mycobacteria were harvested in early to mid log phase of growth and the cells were pelleted at room temperature for $5 \mathrm{~min}$ at $14,000 \mathrm{~g}$. Pellets were resuspended in ATL buffer (Qiagen DNeasy Blood \& Tissue Kit) and the samples were transferred to Lysing
Matrix B tubes $(0.1 \mathrm{~mm}$ silica spheres). Samples were homogenised in a FastPrep ${ }^{\text {TM }}$ FP120 cell disruptor at $3 \times$ 20 s, Speed 6, followed by centrifugation at $14,000 \mathrm{~g}$ for $5 \mathrm{~min}$. Supernatants were transferred to fresh sterile micro centrifuge tubes and Proteinase K (Qiagen DNeasy Kit) was added followed by incubation overnight at $56^{\circ}$ C. DNA was extracted using DNeasy Kit (Qiagen) according to the manufacturer's protocol.

\section{Genome sequencing}

DNA library preparation was carried out by the DNA sequencing teams at the Wellcome Trust Sanger Institute. Multiplexed libraries were sequenced in batches of 12 on the Illumina Genome Analyzer (GAIIx platform) platform to produce $75 \mathrm{bp}$ paired end reads.

\section{SNP analyses and phylogenomics}

The sequencing data were mapped against the Map K10 reference [27] using SMALT (http://sourceforge.net/projects/smalt/) with default parameters. Consensus variants were called using SAMtools and bcftools [28] using filters designed to keep false positives to a minimum, which include a minimum base quality of 50 , a minimum mapping quality of 30 , support from at least 4 reads ( 2 forward and 2 reverse) and an absence of heterozygosity. Previously these filters have been shown to keep the false positive rate to lower than 1 SNP per genome [29]. Variants that passed these filters were used to build a maximum likelihood tree using RAxML v. 7.0.4 [30] with 100 bootstrap replicates.

The BEAST package (v1.7.5), a program for Bayesian Markov chain Monte Carlo (MCMC) analysis of genetic sequences, was used to estimate substitution rates [31]. Input XML files were created using BEAUTi [30] from the whole genome SNP-alignment and the associated dates of isolation for each isolate. For all analyses, three

Table 1 Distance matrix plot showing the number of SNPS present between selected strain groups

\begin{tabular}{lllll}
\hline & Type S & Type S(I) & Type S(III) & Type C \\
\hline Type S & & & & 2360 \\
Type S(I) & & & 1051 & 2684 \\
Type S(III) & & 1051 & & 3087 \\
Type C & 2360 & 2684 & 3087 & \\
Type B & 2565 & 2889 & 3292 & 264 \\
\hline
\end{tabular}




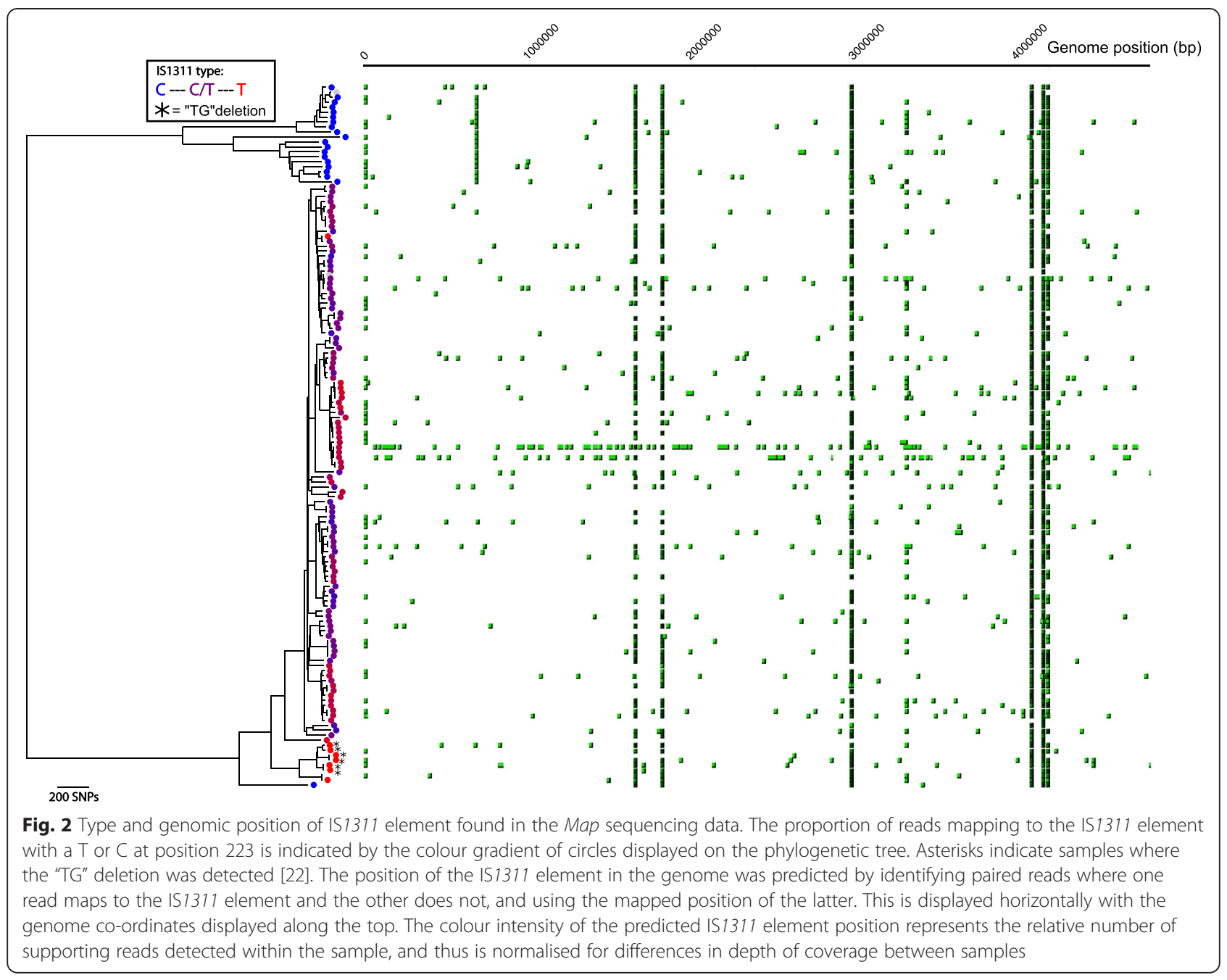

independent MCMC chains of 100 million states were run using a GTR evolutionary model and a variety of combinations of clock and population size models. Tracer (v1.5) [31] was used to assess convergence (after an initial burn-in period of $10 \%$ ), agreement between the three runs and that all effective sample size (ESS) values were greater than 200 .

IS1311 typing was carried out in silico, using the raw sequencing data. All reads were mapped to a representative sequence (locus tag MAP4_RS00055 in reference accession NC_021200.1) using the mapping approach described above, and the proportion of reads mapping to the IS1311 element with a $\mathrm{T}$ or $\mathrm{C}$ at position 223 was counted. The presence of the "TG" deletion [22] was assessed by parsing of the bcf file and manual inspection. The position of the IS1311 elements in the genome of each isolate was predicted by first identifying the corresponding forward/reverse read of the reads identified as matching IS1311 (from above). These were then mapped to the Map K10 reference [27] using SMALT with repeat mapping switched off. The specific position of these reads indicates the position of the IS1311 element.

Publically available Camelid genome sequences [6] were incorporated into the phylogenetic tree by simulating short reads from the de novo assembly, and following the variant calling procedure described above. To do this an in-house script was used to create 75 bp paired reads, assuming a $200 \mathrm{bp}$ insert size in a moving window procedure for every $3 \mathrm{bp}$, resulting in an approximate depth of $25 x$. These simulated reads will not have sequencing errors like normal short-reads, however this approach relies on the accuracy of the original de novo assembly so results should be treated with caution.

\section{Availability of supporting data section}

Raw sequence data supporting the results of this article are deposited in the European Nucleotide Archive (ENA) under accession PRJEB2204. 

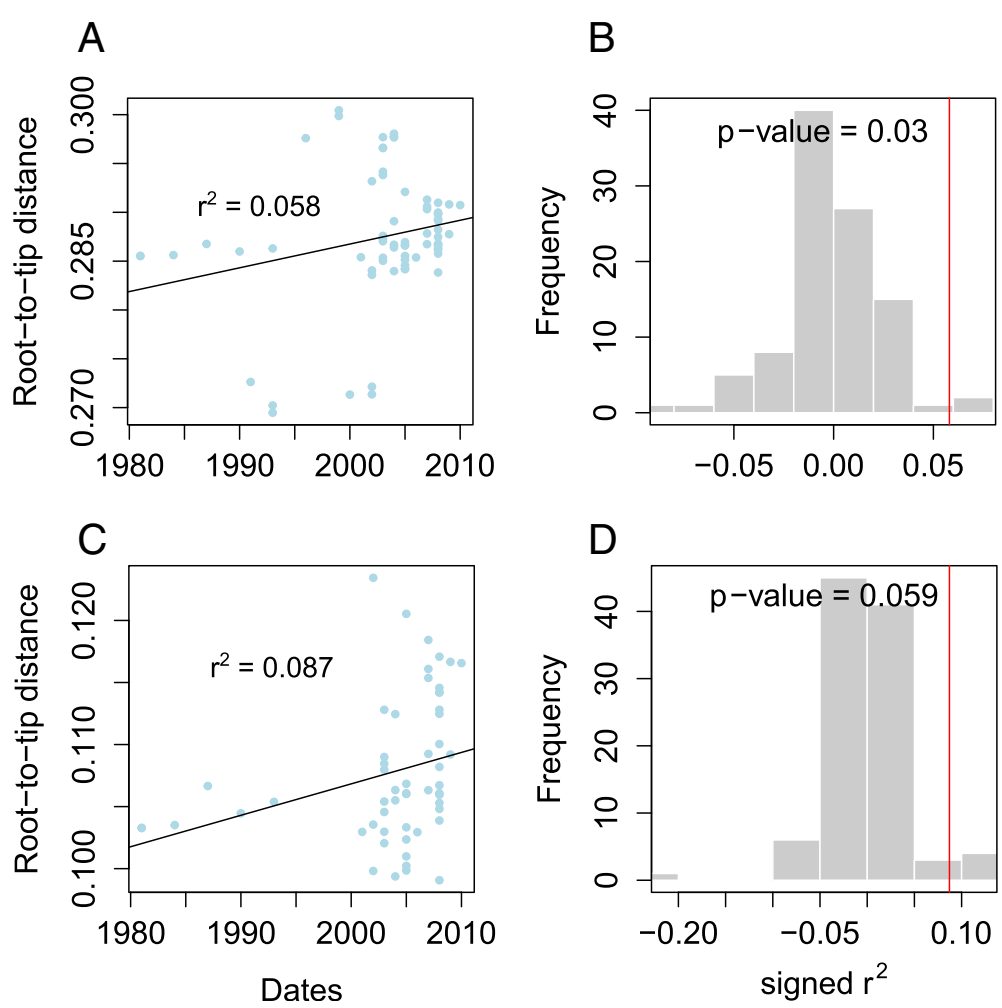

Fig. 3 Investigating the presence of a clock-like signal in the data. The presence of a molecular clock in the dataset was assessed by plotting the root to tip distance of isolates in the phylogeny against isolation date [53] for both the dataset as a whole $(\mathbf{a}+\mathbf{b})$ and only Type II isolates (c $+\mathbf{d})$. Both had evidence of a very weak positive signal, as indicated by a low linear regression correlation coefficient ( $\mathbf{a}+\mathbf{c})$. In order to test the significance of these observations, 99 comparison datasets were produced, in which the isolation dates were permuted on the phylogeny. $\mathbf{b}$ and $\mathbf{d}$ show histograms of correlation coefficient values from the permuted datasets, with the red line indicating the value from the real data. In both cases the correlation coefficient of the real data is significantly greater than the permuted data at the 0.05 level. Passing this test is a minimal requirement for the application of BEAST analysis (Fig. 4), which assumes the presence of a molecular clock

\section{Results and discussion}

\section{Phylogenetic classification of Map isolates}

WGS data were obtained for 141 isolates. The sequences were mapped to the corrected and annotated reference Map strain K10 [27] to identify SNPs. A maximum likelihood phylogenetic tree based on SNPs detected in non-repetitive regions of the genome is depicted in Fig. 1 and Additional file 2: Figure S1. Maa and Mas were included to place the Map strains in a wider mycobacterial context as shown in the inset in the figure. There were more than 40,000 SNPs between the Map isolates and these other members of the Mycobacterium avium complex. SNP distances between the major strain groups are shown in Table 1. The phylogenetic analysis confirmed the division of Map isolates into two major lineages, concordant with the previous Type $\mathrm{S}$ and Type $\mathrm{C}$ designations. We propose that this nomenclature remains for historical reasons but re-emphasise the fact that it does not necessarily reflect host provenance. The Type $\mathrm{S}$ clade included isolates from sheep, goats and deer and the Type $\mathrm{C}$ clade comprised isolates from cattle, sheep, goats, deer, moufflon, bison, buffalo and humans. Type $S$ strains are perceived to have a host preference for sheep and goats but this observation has been largely based on the low frequency of isolation of Type $\mathrm{S}$ strains from other species and could reflect a lack of interaction between the host species rather than a true adaptation to specific host populations. A recent study by Verdugo et al. [32] reported that Type $\mathrm{S}$ strains are more frequent in New Zealand beef cattle than Type $C$ strains where these species are frequently grazed together. Type $C$ strains are isolated from a broad range of hosts and do not appear to have a host preference. The evidence for interspecies transmission is compelling, but the relative risk of transmission of the different strain types between host species cannot be determined with our data. It has been reported that the risk of natural transmission of Type $S$ strains from sheep and goats to cattle is low and occurs only when susceptible animals are exposed to high doses [33].

The phylogenetic analysis clarified the relationship between the previously described Type I and Type III 


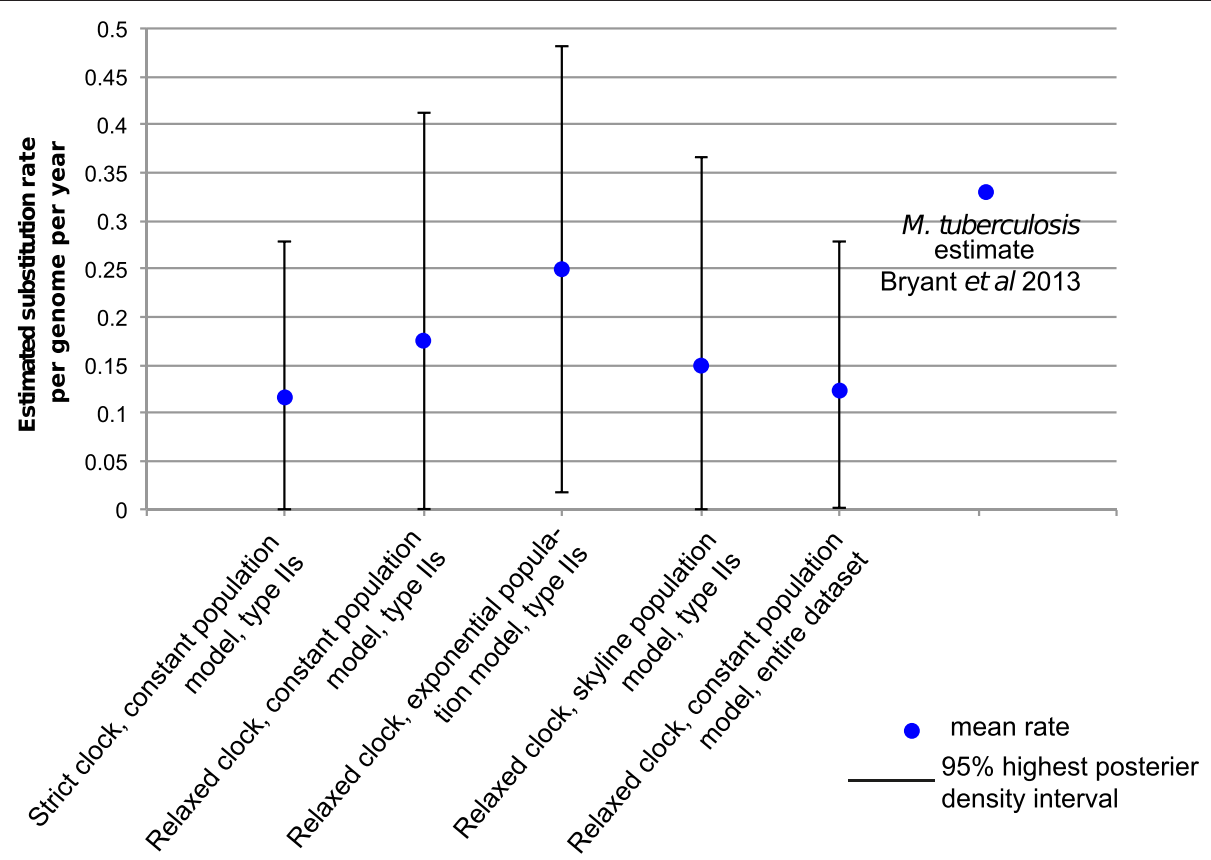

Fig. 4 Estimates of substitution rate. Coalescent analyses were implemented using the BEAST package (v1.7.5) [31] as described in the text. This was carried out both on the entire data and the Type $\mathrm{C}$ isolates alone, using a variety of population models as shown. The mean value represents the mean estimated substitution rate from three independent runs. The confidence intervals represent the maximum and minimum higher posterior densities obtained. As a comparison, the estimated rates are shown alongside a predicted substitution rate of $M$. tuberculosis in the context of transmission [49]

strains. These strain groups are distinct sublineages of Type S, and Type III is not intermediate between the Type $\mathrm{S}$ and Type $\mathrm{C}$ strains as previously suggested. The Type I strains comprise nine of ten pigmented strains but not all strains in this group are pigmented. A New Zealand ovine isolate, MAPMRI161, is not pigmented but is closely related to the Type I group and a Spanish ovine isolate, MAPMRI051, in the Type III group is pigmented. We failed to identify any SNPs, insertions, deletions or the presence or absence of any single gene that could be exclusively associated with pigmented strains. This suggests that the underlying genetic basis for changes in this phenotype is likely to be complex and multi-factorial.

Phylogenetic analysis confirmed that the Type $B$ strains are a subtype of Type $C$ and not restricted to Bison species. The USA Type B isolates could be differentiated from 'Indian bison type' by 255 SNPs and also by PFGE data from this project and data reported previously [21].

The human isolates from Crohn's disease patients did not comprise a distinct phylogenetic group, consistent with previous observations $[34,35]$ but were all Type $C$ strains. There were no SNPs, insertions or deletions found to be exclusive to these isolates. However, although the human isolates did not comprise a single group, we did observe that human isolates from the same country were often closely related (Fig. 1 and Additional file 3: Figure S2). We know of no epidemiological connections between the isolates except for MAPMRI009 and MAPMRI010, which were isolated from the same patient employing different techniques. Furthermore, the human isolates appeared to be closely related to Map isolates from sheep and cattle from the same country, suggesting livestock as a possible source of transmission.

Since this work was initiated, further Map isolates have been sequenced and their genome sequences made available in the public databases. These include the Camelid strains JQ5 and JQ6 [6], S5 Indian bison-type [36], USA Type S isolate S397 [37], Australian ovine isolate CLIJ361 [34], two Australian bovine isolates CLIJ623 and CLIJ644 [34], Australian human isolates Pt139, Pt144, Pt145, Pt146, Pt154, Pt155 and Pt164 [34], USA caprine isolate JTC1285 and an isolate from an oryx JTC1281 [35]. To identify the phylogenetic relationships between these strains and those in our panel, we included these strains in our analysis and the resulting phylogenetic tree is depicted in Additional file 3: Figure $\mathrm{S} 2$. It is clear from the analysis that the available camelid strains represent another subtype of Type S. The S5 Indian bison-type strain clustered with the other non-USA Type B strains confirming the existence of an Indian bison-type group. The USA S397 isolate was most 
closely related to the Type III isolates originating from Spain whereas the Australian ovine isolate CLIJ361 was most closely related to a New Zealand Type I isolate. The remaining isolates were distributed among the Type $\mathrm{C}$ isolates.

In general, there did not appear to be a strong association with geographic location among the isolates in this panel. However, the results are biased in that the isolates were chosen to maximise genetic diversity and have been assembled from a global perspective but with a limited number of isolates from many included countries. WGS of larger numbers of isolates from defined geographic locations may reveal correlations.

\section{Relationship between IS1311 genotype and the whole genome phylogeny}

IS1311 genotyping is used in many laboratories to distinguish between $\mathrm{S}, \mathrm{C}$ and B-type strains. The strains are differentiated on the basis of the number of copies with a $\mathrm{C}$ or a $\mathrm{T}$ at base position 223 in the insertion sequence IS1311 [20]; in this scheme all copies of IS1311 in Btype strains have a $\mathrm{T}$ at position 223 whereas all the Type $\mathrm{S}$ strains have a $\mathrm{C}$ and the Type $\mathrm{C}$ strains have one or more copies with a $\mathrm{C}$ or a $\mathrm{T}$ at the same position. IS1311 genotyping of isolate MAPMRI074 identified the isolate as a Type $S$ strain but its position in the phylogenetic tree placed it as a Type $\mathrm{C}$. This anomaly prompted us to look at the distribution of $\mathrm{C}$ and $\mathrm{T}$ alleles at base pair position 223 for the panel isolates. Reads that mapped to the IS1311 element were identified, and the proportion of base calls for either a $\mathrm{T}$ or $\mathrm{C}$ at nucleotide position 223 was counted as shown in Fig. 2. All Type S strains in the panel have a $C$ at this position and all of the Type $B$ strains have a $T$ as previously reported [20]. All Type B strains had evidence of the "TG" deletion at positions 64 and 65 as previously observed [22]. No differences were observed between the USA bison strains MAPMRI029 and MAPMRI031 and the Indian bisontype group using this assay. However, it is clear that differences exist between the other non-Type B, Type C strains with respect to the number of copies of IS1311 with the T-C allelic variation. The results for some Type C strains not in the Type B cluster suggest that these strains also have the $\mathrm{T}$ allele at base position 223 in all copies of the IS1311 element. As isolate MAPMRI074 was identified as a Type S strain on the basis of an unequivocal result in the IS1311-REA and IS1311 sequence analysis but was clearly a Type $C$ strain confirmed by its positivity for $\mathrm{LSP}^{\mathrm{A}} 20$ and its position within the phylogenetic tree, it suggests that the $\mathrm{C}$ to $\mathrm{T}$ allelic variation at base pair 223 in IS1311 occurred after the initial divergence of Type $\mathrm{C}$ from Type $\mathrm{S}$ strains. This is the first Type C Map isolate to be identified with a Type $\mathrm{S}$ IS1311-REA profile. The isolate did not exhibit any notable phenotypic differences compared with the other Type $\mathrm{C}$ isolates with respect to growth characteristics. These results suggest that the IS1311-REA may not be the best assay for differentiating between Type S, Type $\mathrm{C}$ and Type B. The PCRs for $\mathrm{LSP}^{\mathrm{A}} 20$ and LSP ${ }^{\mathrm{A}} 4$-II [26] may be the better choice for distinguishing Type $\mathrm{C}$ and Type S strains.

\section{Relationship between MIRU-VNTR genotype and the whole genome phylogeny}

MIRU-VNTR genotyping is based on detecting the number of copies of mycobacterial interspersed repetitive units (MIRUs) and variable number tandem repeats (VNTRs) in the genome, which differ between strains. This is probably the most commonly used genotyping method for Map as it is easy to perform, requires only a small amount of DNA and the digital sequence profiles can be stored in databases and compared between laboratories [12]. For this study we used the eight MIRU and VNTR loci described by Thibault and coworkers [24]; MIRUs 292 and X3 and VNTRs 25, 47, 3, 7, 10 and 32. Profile numbers (designated INMV numbers) were assigned by INRA and collated in a publically available database [25]. MIRU-VNTR data available for 105 sequenced isolates were mapped onto the phylogenetic SNP-based tree as shown in Fig. 1 and Additional file 4: Figure S3. A total of 30 different INMV profiles were represented among the isolates.

As expected we observed clear differences in the MIRU-VNTR profile between the Type $S$ and Type $C$ lineages as reported previously by Biet et al. [23]. However, within these lineages MIRU-VNTR provided less discriminatory power, where many isolates shared identical profiles. For example, of the 82 MIRU-VNTR typed Type $C$ strains, 27 and 31 had an identical profile defined as INMV1 and INMV2 respectively, which are the most common profiles across Europe [38] and Canada [11]. Simpson's index of diversity [39] for Type S strains in the panel is 0.7251 and for Type C strains 0.7515 .

Our study strongly supports previous reports that MIRU-VNTR genotyping does not accurately reflect phylogeny and that these repeat sequences are subject to homoplasy [11, 40, 41]. Homoplasy is the occurrence of genotypes that are identical by state but not by descent and can arise by various means including convergent and reverse evolution and horizontal gene transfer. Homoplasy was evident in five of 11 INMV types in which more than one isolate per type was sequenced (INMV 1, 2, 3, 21 and 70). Homoplasy among INMV 2 and 3 was also observed by Ahlstrom et al. [11]. INMV types 21 and 70 were found in both the Type I and Type III sublineages. Hence this first study of a global panel of Map isolates emphasizes that MIRU-VNTR could provide misleading genetic and epidemiological relationships 
between strains and any inference of transmission from identical INMV profiles must be made with caution. In contrast only $2 \%$ of SNPs were observed to be homoplasic, further highlighting the strength of the whole genome approach over MIRU-VNTR typing. This is exemplified by the 12 epidemiologically unrelated isolates with identical combined PFGE ([2-1]), MIRUVNTR (INMV1) profiles, which were included in our study (Additional file 1: Table S1). The phylogenetic analysis showed that these isolates belonged to five different clades within the Type $\mathrm{C}$ lineage (Fig. 1 and Additional file 4: Figure S3), demonstrating that SNP analyses outperform MIRU-VNTR even when the latter is combined with other genotyping techniques.

Genomic changes following continuous passaging in vitro WGS was performed on three passages (P1, P26 and P32 or 37) of strains M21/02, JD143 and MapK10 and the sequences compared to determine any changes in the genome between passages. The field strains M21/02 and JD143 were passaged from primary isolation on 7H11+, P1 representing the first subculture. The MapK10 strain was obtained from the American Type Culture Collection (ATCC BAA 968) and there are no data regarding how many times this strain had been passaged. Therefore, for this strain P1 represents the first passage in our laboratory. For strain M21/02 a SNP $(\mathrm{A} \rightarrow \mathrm{C})$ was detected at position 2041004 at P26 causing a non synonymous mutation in the MAPK_1803 $(g \ln E)$ gene. This SNP was also detected at P37 but no further changes were observed. For JD143, no differences were observed between P1 and P26 but after 37 passages four mutations were detected; two synonymous SNPs were detected at position 812776 in the MAPK_0714 (ligA) gene and at position 4151948 in the MAPK_3716 $(g \ln Q)$ gene and two non synonymous SNPs at position 1488559 within MAPK_1342 (a pseudogene) and at 1687561 in MAPK_1510 (nadE). For MapK10 an insertion of a $\mathrm{G}$ at position 4410158 within MAPK_3949 was detected at P26, which causes a frameshift in mmpL4_7. This mutation was also present at P32.

It is interesting that so many of these mutations affect genes involved in glutamine pathways for nitrogen assimilation. Glutamine and glutamate are the two major amino acids that act as cellular nitrogen donors for the synthesis of biomolecules within the cell [42]. In mycobacteria, the assimilation of inorganic nitrogen and its conversion to glutamine and glutamate is carried out by glutamine synthetase whose activity is modulated by GlnE, an adenylyl transferase [43]. NadE is a glutaminedependent $\mathrm{NAD}(+)$ synthetase that obtains ammonia through the hydrolysis of glutamine to glutamate and GlnQ is a glutamine $\mathrm{ABC}$ transporter ATP-binding protein. GlnE is essential for growth of M.tuberculosis [44] whereas GlnQ is a non-essential gene and is in fact deleted in some clinical M.tuberculosis isolates. It is tempting to suggest that the mutations arising in these genes in the two field strains are adaptations to the growth medium. The Map field isolates were propagated on $7 \mathrm{H} 11+$, which contains ammonium sulphate, Lglutamate, ferric ammonium citrate and L-asparagine as sources of nitrogen. Under these conditions, nitrogen is not a limiting factor and the cells would not need to expend energy acquiring nitrogen. It is known that media constituents can modulate aerobic expression of genes and operons, for example, devR-devS [45]. In addition, genomic changes in Map potentially arising as a result of long term growth on potato starch medium and Dubos medium with added pyruvate have been reported [46]. Interestingly, Hsu et al. [35] reported a SNP in the $g \ln E$ gene in all six Map genomes sequenced and postulated that this might be an indication of common evolutionary ancestor with environmental isolates. It is possible that the $g \ln E$ gene may be a hotspot for mutation in the genome allowing for rapid adaptation to niche-specific conditions.

The frameshift mutation within mmpL4_7 in MapK10 is also interesting. Inactivation of $m m p L$ genes typically leads to a change in surface characteristics, which could include altered colony morphology, reduced sliding motility and reduced biofilm formation. These characteristics were not observed on the $7 \mathrm{H} 11+$ slopes during this study. The $m m p L$ genes are involved in virulence in M.tuberculosis [47]. MmpL4 is required for optimal growth of M.tuberculosis and survival in mouse lungs [47] and is down regulated during nutrient starvation [48]. Therefore, a mutation in MmpL4_7 potentially could reduce the virulence of the MapK10 strain.

\section{Estimation of the substitution rate in Map}

We investigated the presence of a molecular clock in the dataset by plotting the root to tip distance of every isolate in the phylogeny against the date of their isolation. This was carried out on the dataset as a whole (where the date of isolation was available) as well as on only Type-C isolates, using a variety of different evolutionary and population models. A weak positive signal was detected (Fig. 3a, c), providing weak evidence for the presence of a molecular clock. However, when this signal was compared to those obtained from trees where isolation date was randomized, this relationship was found to be statistically significant for the entire dataset but not Type Cs in isolation (Fig. 3b, d), which provides support for the presence of a molecular clock in this dataset. Coalescent analysis implemented in BEAST was used to estimate the substitution rate in Map (Fig. 4). All estimates agreed on a low substitution rate, with an 
upper limit of less than half a SNP per genome per year. Considering the average mutation rate of 0.3 SNPs per genome per year estimated for M.tuberculosis [49] and the longer doubling time of 22-26 h for Map [50] compared with $16 \mathrm{~h}$ for M.tuberculosis in optimal laboratory conditions [51], a lower average mutation rate would be consistent for Map

The low sequence diversity and substitution rate for Map has implications for epidemiological investigations tracing the sources of infections. Even with the ultimate resolution afforded by WGS, this alone will not be sufficient to track transmission or reconstruct outbreaks. However, constructing phylogenetic trees from SNP data could help to determine if a recent transmission event has occurred. For example, isolates that represent recent transmission events would be expected to be located adjacent to one another on the tree and share a common ancestor. Regardless of WGS data, epidemiological data will be paramount for determining Map transmission events, more so than for faster evolving pathogens.

\section{Conclusions}

This study has clarified the phylogenetic relationships between the different Map strain groups that have been reported in the literature. There are two major lineages concordant with the previously described Type $\mathrm{S}$ and Type $\mathrm{C}$ designations. The Type I and Type III strain groups are subtypes of Type $\mathrm{S}$. The pigmented strains belonged to the Type $\mathrm{S}$ lineage but did not comprise a distinct clade in the phylogenetic tree. Although phenotypically distinct from other strains, we could not identify any SNPs, insertions, deletions or the presence or absence of any single gene that could be exclusively associated with pigmented strains. Type B strains are a subtype of Type $C$ and not restricted to Bison species. The Indian bison-type is a subtype of Type B. As more isolates are sequenced, more genetic polymorphisms will be identified and the phylogenetic tree will require updating. A central public repository of Map genome sequences and corresponding metadata (particularly epidemiological, pathological and virulence data) would greatly facilitate future studies.

With the dataset employed in this study, there was no evidence for strong geographical clustering. The data supports the perception that Type S strains have a preference for sheep and goats suggesting host adaptation may have occurred but this could be biased by the selection of isolates available for study. The human isolates from Crohn's patients did not comprise a distinct single group but isolates from the same country were often closely related to one another and other Map isolates from sheep and cattle from the same country, suggesting livestock as a possible source of transmission.
Overall, the Map isolates exhibited restricted genetic diversity and estimates of the substitution rate were less than half a SNP per genome per year, which presents a significant challenge for genotyping and epidemiological tracing. The study highlights the limitations of two commonly used genotyping methods, IS1311 typing and MIRU-VNTR. IS1311 typing is used to differentiate between Types $\mathrm{S}, \mathrm{C}$ and $\mathrm{B}$, but this study suggests that the allelic variation at base pair 223 in IS1311 used for discrimination occurred after the initial divergence of Type $\mathrm{C}$ from Type $\mathrm{S}$ strains and does not assign all of the studied strains to the correct lineage. MIRU-VNTR provides more discriminatory power than IS1311 typing and will differentiate between Type $S$ and Type $C$ but provides limited resolution within these lineages. The polymorphisms detected by MIRU-VNTR do not necessarily accurately reflect the phylogenetic relationships between strains because strains with identical MIRU-VNTR profiles may be distantly related and the data should be treated with caution. WGS provides the ultimate resolution of different isolates and is much more informative than standard genotyping methods. However, WGS alone will not be sufficient for tracing and tracking Map infections, yet importantly it can provide a phylogenetic context for affirming epidemiological connections.

WGS of strains passaged in vitro has highlighted the mutability of the Map genome and how relatively quickly mutations can be selected under different environmental conditions. This emphasizes the need to minimize the subculture of Map strains and to use lowpassage strains for studies wherever possible.

In summary, this study clarifies the phylogenetic relationships between the previously described Map strain groups, and highlights the limitations of current genotyping techniques. WGS of additional isolates will identify further phylogenetic relationships and help to resolve the epidemiology, evolution, population structure and phylogeography of Map.

\section{Additional files}

Additional file 1: Table S1. Information on Map strains included in this study (XLSX $23 \mathrm{~kb}$ )

Additional file 2: Figure S1. Maximum likelihood phylogenetic tree of Map strains sequenced in this study. The tree was based on the SNPS identified through mapping to Map K10 as described in the text and built using RAxML v. 7.0.4 [30] with 100 bootstrap replicates. Branches are annotated with bootstrap values and the tips with the isolate MAPMRI numbers. (PDF $33 \mathrm{~kb}$ )

Additional file 3: Figure S2. Phylogenetic tree of sequenced and publically available Map strains. The data from this study were combined with previously published WGS Map data (in red) and the phylogenetic analysis was repeated. The publically available sequences were obtained from $[6,34-37]$. At the time of analysis the raw sequencing data was not available for the Camelid strains so short $75 \mathrm{bp}$ paired reads were simulated from the assembled contiguous sequences. (PDF 38 kb) 
Additional file 4: Figure S3. Comparison between SNP-based phylogeny and MIRU-VNTR and INMV types. The phylogenetic tree presented in Fig. 1 is shown alongside the MIRU-VNTR and INMV types, where colours represent versions of the loci as indicated in the legend. Spaces represent missing data. Dashed lines represent the Map strains with identical PFGE/MIRU-VNTR profiles ([2-1]1). (PDF $37 \mathrm{~kb})$

\section{Competing interests}

The authors declare that they have no competing interests.

\section{Authors' contributions}

$J M B, S R H$ and VCT carried out the SNP and phylogenetic analyses, data interpretation and helped draft the manuscript. VCT, JMCL, IH, IAS and FB performed the IS1311, PFGE, MIRU-VNTR, propagation and selection of strains and DNA preparation for WGS. DGES, DJM, SJB, JP and KS conceived and designed the study. DGES and KS participated in data interpretation and drafting the manuscript. All authors read and approved the final manuscript.

\section{Acknowledgements}

The authors would like to thank the following colleagues/organizations for contributing strains to this project: F Saxegaard, S Nilsen, TB Johansen (National Veterinary Institute, Oslo, Norway), D Bakker (Central Veterinary Institute, Lelystad, Netherlands), P Overduin (Netherlands National Institute for Public Health and the Environment [RIVM], Netherlands), Z Dimareli-Malli (Veterinary Research Institute of Thessaloniki, National Agricultural Research Foundation, Thessaloniki, Greece), P Kostoulas (University of Thessaly, Greece), I Pavlik (National Veterinary Institute, Brno, Czech Republic), N Arrigoni (Istituto Zooprofilattico Sperimentale della Lombardia e del I'Emilia Romagna - Sezione di Piacenza, Italy), N Pozzato (Istituto Zooprofilattico Sperimentale delle Venezie, Verona, Italy), P Möbius (Friedrich-Loeffler-Institut [FLI], Jena, Germany, BN Tripathi (Indian Veterinary Research Institute, Izatnagar, India), DM Collins (AgResearch, Limited, Upper Hutt, New Zealand), G Thomas (University of Wisconsin School of Veterinary Medicine, Wisconsin, USA), J O'Mahony (Cork Institute of Technology, Cork, Republic of Ireland), I Grant (Queen's University, Belfast, Northern Ireland, UK), R Pickup (Lancaster University, Lancaster, UK), G Caldow, D Henderson (Scotlands Rural College [SRUC], Scotland, UK), M Cranwell, A Holliman (Animal Health Veterinary Laboratories Agency, England, UK). This project was supported by the Scottish Government Rural and Environment Science and Analytical Services Division (KS, JM, IH, DGES), Wellcome Trust grant No. 098051 (JMB, SRH, JP), the European Commission Marie Curie IEF Grant Agreement No. PIEF-GA-2009-251953 (VCT), the Institut National de la Recherche Agronomique (INRA) (FB) and the Área de Agricultura, Pesca y Política Alimentaria of the Departamento de Desarrollo Económico y Competitividad of the Basque Government (IAS).

\section{Author details}

${ }^{1}$ Wellcome Trust Sanger Institute, Genome Campus, Cambridge, UK. ${ }^{2}$ Division of Infection and Immunity, University College London, London, UK. ${ }^{3}$ Moredun Research Institute, Pentlands Science Park, Penicuik EH26 OPZ, UK. ${ }^{4}$ Institute of Infection, Immunity \& Inflammation, University of Glasgow, Glasgow G12 8QQ, UK. ${ }^{5}$ Neiker-tecnalia, Dpto. de Producción y Sanidad Animal, Berreaga 1, 48160 Derio, Bizkaia, Spain. ${ }^{6}$ INRA, UMR1282, Infectiologie Santé Publique (ISP-311), F-37380 Nouzilly, France. ${ }^{7}$ Department of Veterinary Medicine, University of Cambridge, Cambridge, UK.

Received: 27 February 2015 Accepted: 5 October 2015

Published online: 26 January 2016

\section{References}

1. Barkema HW, Hesselink JW, McKenna SLB, Benedictus G, Groenendaal H. Global prevalence and economics of infection with Mycobacterium avium subsp. paratuberculosis in ruminants. In: Behr MA, Collins DM, editors. Paratuberculosis: organism, disease, control. Cambridge: CAB International; 2010. p. 10-21.

2. Motiwala AS, Amonsin A, Strother M, Manning EJ, Kapur V, Sreevatsan S. Molecular epidemiology of Mycobacterium avium subsp. paratuberculosis isolates recovered from wild animal species. J Clin Microbiol. 2004;42:1703-12.

3. Hutchings MR, Stevenson K, Greig A, Davidson R, Marion G, Judge J. Infection of non-ruminant wildlife by Mycobacterium avium subsp. paratuberculosis. In: Behr MA, Collins DM, editors. Paratuberculosis: organism, disease, control. Cambridge: CAB International; 2010. p. 188-200.
4. Ghadiali AH, Strother M, Naser SA, Manning EJ, Sreevatsan S. Mycobacterium avium subsp. paratuberculosis strains isolated from Crohn's disease patients and animal species exhibit similar polymorphic locus patterns. J Clin Microbiol. 2004;42:5345-8.

5. Clarke CJ. The pathology and pathogenesis of paratuberculosis in ruminants and other species. J Comp Pathol. 1997;116:217-61.

6. Ghosh P, Hsu C, Alyamani EJ, Shehata MM, Al-Dubaib MA, Al-Naeem A, et al. Genome-wide analysis of the emerging infection with Mycobacterium avium subspecies paratuberculosis in the Arabian camels (Camelus dromedarius). PLoS One. 2012;7:e31947.

7. Mackintosh C, Griffin JF. Paratuberculosis in deer, camelids and other ruminants. In: Behr MA, Collins DM, editors. Paratuberculosis: organism, disease, control. Cambridge: CAB International; 2010. p. 179-87.

8. Beard PM, Rhind S, Buxton D, Daniels MJ, Henderson D, Pirie A, et al. Natural paratuberculosis infection in rabbits in Scotland. J Comp Pathol. 2001;124: 290-9.

9. Salgado M, Monti G, Sevilla I, Manning E. Association between cattle herd Mycobacterium avium subsp. paratuberculosis (MAP) infection and infection of a hare population. Trop Anim Health Prod. 2014;46:1313-6.

10. Naser SA, Sagramsingh SR, Naser AS, Thanigachalam S. Mycobacterium avium subspecies paratuberculosis causes Crohn's disease in some inflammatory bowel disease patients. World J Gastroenterol. 2014;20:7403-15.

11. Ahlstrom C, Barkema HW, Stevenson K, Zadoks RN, Biek R, Kao R, et al. Limitations of variable number of tandem repeat typing identified through whole genome sequencing of Mycobacterium avium subsp. paratuberculosis on a national and herd level. BMC Genomics. 2015;16:161.

12. Collins DM. Strain characterization of Mycobacterium avium subsp. paratuberculosis. In: Behr MA, Collins DM, editors. Paratuberculosis: organism, disease, control. Cambridge: CAB International; 2010. p. 294-305.

13. Stevenson K. Comparative differences between strains of Mycobacterium avium subsp. paratuberculosis. In: Behr MA, Collins DM, editors. Paratuberculosis: organism, disease, control. Cambridge: CAB International; 2010. p. 126-37.

14. Collins DM, Gabric DM, de Lisle GW. Identification of two groups of Mycobacterium paratuberculosis strains by restriction endonuclease analysis and DNA hybridization. J Clin Microbiol. 1990;28:1591-6.

15. Stevenson $K$, Hughes VM, de Juan L, Inglis NF, Wright F, Sharp JM. Molecular characterization of pigmented and non-pigmented isolates of Mycobacterium avium subspecies paratuberculosis. J Clin Microbiol. 2002;40: 1798-804.

16. Stevenson K. Genetic diversity of Mycobacterium avium subspecies paratuberculosis and the influence of strain type on infection and pathogenesis: a review. Vet Res. 2015;46:64.

17. de Juan L, Mateos A, Domínguez L, Sharp JM, Stevenson K. Genetic diversity of Mycobacterium avium subspecies paratuberculosis isolates from goats detected by pulsed-field gel electrophoresis. Vet Microbiol. 2005;106:249-57.

18. Castellanos E, Alvarez J, Aranaz A, Romero B, De Juan L, Bezos J, et al. Use of Single Nucleotide Polymorphisms in inh-A gene to characterize Mycobacterium avium subsp. paratuberculosis into Types I, II and III. In: Nielsen SS, editor. Proceedings of the Ninth International Colloquium on Paratuberculosis: 29 October - 2 November 2007; Tsukuba. Tsukuba: International Association for Paratuberculosis; 2007. p. 6-8.

19. Castellanos E, Aranaz A, Romero B, de Juan L, Àlvarez J, Bezos J, et al. Polymorphisms in gyrA and gyrB genes among Mycobacterium avium subsp. paratuberculosis Type I, II, and III isolates. J Clin Microbiol. 2007;45:3439-42.

20. Whittington RJ, Marsh IB, Whitlock RH. Typing of IS1311 polymorphisms confirms that bison (Bison bison) with paratuberculosis in Montana are infected with a strain of Mycobacterium avium subsp paratuberculosis distinct from that occurring in cattle and other domesticated livestock. Mol Cell Probes. 2001;15:139-45.

21. Sevilla I, Singh SV, Garrido JM, Aduriz G, Rodríguez S, Geijo MV, et al. Molecular typing of Mycobacterium avium subsp. paratuberculosis strains from different hosts and regions. Sci Tech Rev (OIE). 2005;24:1061-6.

22. Sohal JS, Singh SV, Singh PK, Singh AV, Kumar N. A new marker IS1311 L2 PCR-REA for identification of 'Indian Bison' type Mycobacterium avium subspecies paratuberculosis. Indian J Biotechnol. 2013;12:204-7.

23. Biet F, Sevilla IA, Cochard T, Lefrançois LH, Garrido JM, Heron I, et al. Inter and intra-subtype genotypic differences that differentiate Mycobacterium avium subsp. paratuberculosis strains. BMC Microbiol. 2012;12:264.

24. Thibault VC, Grayon M, Boschiroli ML, Hubbans C, Overduin P, Stevenson K, et al. New variable number tandem repeat markers for typing 
Mycobacterium avium subsp. paratuberculosis and Mycobacterium avium strains: comparison with IS900 RFLP and IS1245 RFLP typing. J Clin Microbiol. 2007:45:2404-10.

25. Mac INMV database. [http://mac-inmv.tours.inra.fr].

26. Semret M, Turenne CY, de Haas P, Collins DM, Behr MA. Differentiating hostassociated variants of Mycobacterium avium by PCR for detection of large sequence polymorphisms. J Clin Microbiol. 2006:44:881-7.

27. Wynne JW, Seeman T, Bulach D, Coutts SA, Talaat AM, Michalski WP. Re-sequencing the Mycobacterium avium subspecies paratuberculosis K10 genome: improved annotation and revised genome sequence. J Bacteriol. 2010;192:6319-20.

28. Li H, Handsaker B, Wysoker A, Fennell T, Ruan J, Homer N, et al. The Sequence Alignment/Map format and SAMtools. Bioinformatics. 2009;25: 2078-9.

29. Harris SR, Feil EJ, Holden MT, Quail MA, Nickerson EK, Chantratita N, et al. Evolution of MRSA during hospital transmission and intercontinental spread. Science. 2010;327:469-74.

30. Stamatakis A. RAxML-VI-HPC: maximum likelihood-based phylogenetic analyses with thousands of taxa and mixed models. Bioinformatics. 2006;22: 2688-90.

31. Drummond AJ, Rambaut A. BEAST: Bayesian Evolutionary Analysis by Sampling Trees. BMC Evol Biol. 2007;7:214.

32. Verdugo C, Pleydell E, Price-Carter M, Prattley D, Collins D, de Lisle G, et al. Molecular epidemiology of Mycobacterium avium subsp. paratuberculosis isolated from sheep, cattle and deer on New Zealand pastoral farms. Prev Vet Med. 2014;117:436-46.

33. Moloney BJ, Whittington RJ. Cross species transmission of ovine Johne's disease from sheep to cattle: an estimate of prevalence in exposed susceptible cattle. Aust Vet J. 2008:86:117-23.

34. Wynne JW, Bull TJ, Seeman T, Bulach DM, Wagner J, Kirkwood CD, et al. Exploring the zoonotic potential of Mycobacterium avium subspecies paratuberculosis through comparative genomics. PLoS One. 2011;6:e22171.

35. Hsu C-Y, Wu C-W, Talaat AM. Genome-wide sequence variation among Mycobacterium avium subspecies paratuberculosis isolates: a better understanding of Johne's disease transmission dynamics. Front Microbiol. 2011;2:236.

36. Singh SV, Kumar N, Singh SN, Bhattacharya T, Sohal JS, Singh PK, et al. Genome sequence of the "Indian Bison Type" biotype of Mycobacterium avium subsp. paratuberculosis strain S5. Genome Announc. 2013;1(1): e00005-13.

37. Bannantine JP, Wu C-W, Hsu C, Zhou S, Schwartz DC, Bayles DO, et al. Genome sequencing of ovine isolates of Mycobacterium avium subspecies paratuberculosis offers insights into host association. BMC Genomics. 2012; 13:89.

38. Stevenson K, Àlvarez J, Bakker D, Biet F, de Juan L, Denham S, et al. Occurrence of Mycobacterium avium subspecies paratuberculosis across host species and European countries with evidence for transmission between wildlife and domestic ruminants. BMC Microbiol. 2009;9:212.

39. Hunter PR, Gaston MA. Numerical index of the discriminatory ability of typing systems - an application of Simpson's index of diversity. I Clin Microbiol. 1988;26:2465-6.

40. Comas I, Homolka S, Niemann S, Gagneux S. Genotyping of genetically monomorphic bacteria: DNA sequencing in Mycobacterium tuberculosis highlights the limitations of current methodologies. PLoS One. 2009;4(11): e7815.

41. Reyes J, Chan CHS, Tanaka MM. Impact of homoplasy on variable numbers of tandem repeats and spoligotypes in Mycobacterium tuberculosis. Infect Genet Evol. 2012;12:811-8.

42. Newsholme P, Procopio J, Lima MM, Pithon-Curi TC, Curi R. Glutamine and glutamate -their central role in cell metabolism and function. Cell Biochem Funct. 2003;21:1-9.

43. Carroll P, Pashley CA, Parish T. Functional analysis of $\mathrm{GlnE}$, an essential adenylyl transferase in Mycobacterium tuberculosis. J Bacteriol. 2008;190: 4894-902

44. Parish T, Stoker NG. glnE is an essential gene in Mycobacterium tuberculosis. J Bacteriol. 2000;182:5715-20

45. Malhotra V, Tyagi JS, Clark-Curtiss JE. DevR-mediated adaptive response in Mycobacterium tuberculosis H37Ra: links to asparagine metabolism. Tuberculosis (Edinb). 2009;89:169-74.
46. Bull TJ, Schock A, Sharp JM, Greene M, McKendrick IJ, Sales J, et al. Genomic variations associated with attenuation in Mycobacterium avium subsp. paratuberculosis vaccine strains. BMC Microbiol. 2013;13:11.

47. Domenech P, Reed MB, Barry 3rd CE. Contribution of the Mycobacterium tuberculosis $\mathrm{MmpL}$ protein family to virulence and drug resistance. Infect Immun. 2005;73:3492-501.

48. Betts JC, Lukey PT, Rob LC, MCAdam RA, Duncan K. Evaluation of a nutrient starvation model of Mycobacterium tuberculosis persistence by gene and protein expression profiling. Mol Microbiol. 2002;43:717-31.

49. Bryant JM, Schürch AC, van Deutekom H, Harris SR, de Beer JL, de Jager V, et al. Inferring patient to patient transmission of Mycobacterium tuberculosis from whole genome sequencing data. BMC Infect Dis. 2013;13:100.

50. Bannantine JP, Zhang Q, Li LL, Kapur V. Genomic homogeneity between Mycobacterium avium subsp. avium and Mycobacterium avium subsp. paratuberculosis belies their divergent growth rates. BMC Microbiol. 2003:3:10

51. Beste DJ, Espasa M, Bonde B, Kierzek AM, Stewart GR, McFadden J. The genetic requirements for fast and slow growth in mycobacteria. PLoS One. 2009;4(4)::5349.

52. Price MN, Dehal PS, Arkin AP. FastTree: computing large minimum evolution trees with profiles instead of a distance matrix. Mol Biol Evol. 2009;26:1641-50

53. Firth C, Kitchen A, Shapiro B, Suchard MA, Holmes EC, Rambaut A. Using time-structured data to estimate evolutionary rates of double-stranded DNA viruses. Mol Biol Evol. 2010;27:2038-51.

\section{Submit your next manuscript to BioMed Central and take full advantage of:}

- Convenient online submission

- Thorough peer review

- No space constraints or color figure charges

- Immediate publication on acceptance

- Inclusion in PubMed, CAS, Scopus and Google Scholar

- Research which is freely available for redistribution 